\title{
A Perfect Storm? Health Anxiety, Contamination Fears, and COVID-19: Lessons Learned from Past Pandemics and Current Challenges
}

\author{
Danielle Dennis $^{1,2}$ (D) Cynthia Radnitz ${ }^{1} \cdot$ Michael G. Wheaton ${ }^{2}$
}

Accepted: 15 April 2021/ Published online: 22 April 2021

(C) Springer Nature Switzerland AG 2021, corrected publication 2021

\begin{abstract}
The novel coronavirus disease 2019 (COVID-19) rapidly spread, becoming a global pandemic with significant health, economic, and social impacts. COVID-19 has caused widespread anxiety, which at healthy levels leads to adaptive, protective behavioral changes. For some individuals, a pandemic outbreak can lead to excessive, maladaptive levels of anxiety, particularly among those with obsessive-compulsive disorder (OCD) and health anxiety. In the present paper, we review past research studies that examined anxiety in response to other disease outbreaks (including Swine Flu, Zika, and Ebola) to serve as a guide for expectable responses to COVID-19. Our review focused on the role of belief-based cognitive variables (obsessive beliefs, contamination cognitions), transdiagnostic processes (disgust sensitivity, anxiety sensitivity, an intolerance of uncertainty), social factors, and environmental/situational variables as contributing factors to excessive concerns about past pandemics. These factors in combination with unique characteristics of the virus (disease, behavioral, social and economic factors) and media consumption might enhance vulnerability to excessive anxiety about COVID-19, in line with a diathesis-stress model. COVID-19 is also unique from past pandemics due to its severity, easy transmissibility, and the nature of prescribed behavioral responses (i.e., hand washing and social distancing). We therefore discuss the ways in which COVID-19 may disproportionately affect individuals with OCD and health anxiety. We conclude with important topics for clinical and research attention to help mental health professionals respond in this time of crisis.
\end{abstract}

Keywords COVID-19 $\cdot$ Health anxiety $\cdot$ Contamination $\cdot$ Pandemic $\cdot$ OCD

Danielle Dennis

dbd2110@student.fdu.edu

1 Fairleigh Dickinson University, Teaneck, NJ, USA

2 Barnard College of Columbia University, New York City, NY, USA 


\section{Introduction}

The novel coronavirus disease 2019 (COVID-19) was first reported in China on December 12, 2019, as causing severe acute respiratory infection (Wuhan Municipal Health Commission, 2019). SARS-CoV-2, the virus that causes COVID-19, comes from a large family of viruses known to cause illnesses including the common cold as well as serious and lethal diseases such as Middle East Respiratory Syndrome (MERS) and Severe Acute Respiratory Syndrome (SARS). As COVID-19 has become a global pandemic with escalating infection and death rates as well as widespread social disruption, there has been a parallel increase in psychological problems (Asmundson \& Taylor, 2020). Research into health anxiety and contamination concerns in past pandemics can provide insight into the nature of anxiety symptoms and related behaviors that may accompany the current COVID-19 pandemic. At the same time, unique aspects of the current COVID-19 outbreak differentiate it from past pandemics. These characteristics should be considered in both clinical work with patients experiencing COVID-related fears and in future research into the psychological effects of the outbreak.

\section{Anxiety, OCD, and Pandemics IIInesses}

Anxiety is often an adaptive emotion, as it may focus attention and motivate important actions when a danger is present. Anxiety about one's health in particular can promote behaviors geared toward preserving health and well-being (Taylor \& Asmundson, 2004). However, health anxiety can become problematic when the anxiety is chronic and excessive or when it is significantly greater than the severity of the health-related threat (Taylor et al., 2012). Therefore, it is worth considering the ways the psychological impacts of COVID-19 may range from helpful and adaptive anxiety to excessive interfering maladaptive anxiety.

Individuals with pre-existing anxiety-related conditions, including obsessivecompulsive disorder (OCD), may be particularly susceptible to developing excessive, maladaptive anxiety during a pandemic outbreak. OCD is a heterogeneous disorder that involves intrusive and unwanted thoughts known as obsessions that are alleviated by ritualistic overt actions or internal neutralizing conditions known as compulsions (American Psychological Association, 2013). Compulsive washing and contamination fear are among the most common symptoms of OCD (Jones \& Menzies, 1997), and conceptual models of OCD consistently find contamination obsessions and washing/ cleaning compulsions to be a distinct symptom dimension (McKay et al., 2004). Contamination fears in OCD are often motivated by harm avoidance related to fears of contracting or spreading diseases (Riskind, 1997). Therefore, pandemic illness threats would be particularly salient for OCD patients with these symptoms.

Similarly, pandemic illness outbreaks are relevant threats to individuals with severe health anxiety (SHA), which has been characterized as a debilitating and persistent fear of having or acquiring a serious disease (Taylor \& Asmundson, 2004). Individuals with health anxiety perceive bodily sensations such as coughing or aching muscles as symptoms of being sick (Asmundson et al., 2010). Although SHA and OCD are different constructs that share many similar features, some authors have suggested that SHA belongs among a spectrum of OCD-related conditions (Murphy et al., 2010). 
Individuals with both SHA and OCD overestimate the likelihood of threat, which leads to an increase in anxiety levels, often prompting checking behaviors (Abramowitz \& Braddock, 2006). Furthermore, both OCD and SHA are associated with difficulty dealing with ambiguity and uncertainty (Deacon \& Abramowitz, 2008).

Considering that the full extent of the COVID-19 pandemic remains unknown given that the crisis is still unfolding, studies that reported on past pandemics can provide valuable insights into how anxiety and OCD symptoms might occur in response to COVID-19. Therefore, in this narrative review, we examined articles that reported on the psychological effects of past outbreaks, including SARS, H1N1 (Swine Flu), Zika virus, Ebola virus, and HIV/AIDs, as responses to these past outbreaks may shed light on expectable responses to COVID-19.

\section{Overview of Recent Pandemics}

Several notable pandemics have spread across the globe in recent history. The spread of the H1N1 "Swine Flu" was first detected in April of 2009, and by June, the World Health Organization had declared it a pandemic (Triana-Baltzer et al., 2009). Media reports at the time compared this outbreak to the "Spanish Flu" of 1918, which killed over 50 million individuals (Johnson \& Mueller, 2002), although ultimately, the death toll was much less (Watanabe \& Kawaoka, 2011).

The Zika virus was originally discovered in 1947 in Uganda and is typically transmitted to humans via a bite from an infected mosquito (Hills et al., 2016). The most common symptoms of Zika are rash, joint pain, and conjunctivitis, but the biggest concern is vertical transmission from pregnant women to their fetuses causing the children to develop microcephaly. Aside from the risk to the unborn fetus, symptoms do not usually persist for more than 7 days (CDC, 2016) and the mortality rate is extremely low.

The outbreak of an unusual and highly contagious pneumonia known as SARS caused panic in the Far East due to its high transmission and mortality rates. This outbreak caused fear in both hospital workers and the general public (Maunder et al., 2003). In Beijing, most work was at a standstill, schools closed classes, the public rushed to buy scarce resources, college students returned home from campus, and many people delayed treatment for other conditions to avoid going to the hospital. The first case of SARS was discovered in December 2002, and the disease was controlled in June 2003. There were 8641 confirmed cases with a death toll of 804 individuals.

Ebola was first discovered in the Democratic Republic of the Congo and is a rare but deadly animal-borne disease transmitted through direct contact with contaminated objects or bodily fluids. The CDC had chronicled 35 Ebola virus outbreaks between 1976 and 2014 (CDC, 2014). The 2014 West Africa outbreak is considered the largest, with at least 21,000 affected human cases totaling nearly 9000 deaths worldwide. Additionally, there was a single confirmed Ebola related death in the USA during 2014.

First identified in 1981, acquired immune deficiency syndrome (AIDS) is the last stage of an infection of human immunodeficiency virus (HIV) which targets and damages the immune system (Deeks, Overbaugh, Phillips, \& Buchbinder, 2015). HIV attacks cells that help fight infection, which makes individuals with HIV more vulnerable to other infections. It is spread through direct contact with bodily fluids, such as through injection with infected needles or via unprotected sexual intercourse. 


\section{Psychological Constructs Linked to Past Pandemics}

A review of the literature on psychological responses to past pandemics via the electronic databases PsycINFO, MEDLINE, ScienceDirect, and PubMed was conducted to examine the role of psychological diatheses (e.g., cognitive variables) as well as stress (e.g., environmental experiences). We organized the results into four separate themes, including belief-based cognitive variables, transdiagnostic cognitive processes, social, and environmental/situational variables. Conceptually, these factors can be considered within a diathesis-stress model, in which certain variables exist as vulnerability factors which in concert with proximal factors may lead to excessive anxiety during pandemics.

\section{Belief-Based Cognitive Variables}

Consistent with Beck's (1979) cognitive approach to emotional disorders, which suggests that maladaptive emotions and behaviors result from dysfunctional beliefs and interpretations (i.e., cognitions), certain cognitive variables have been associated with anxiety in response to past pandemics. For example, Brand et al. (2013) found that scores on the Obsessive Beliefs Questionnaire (Obsessive Compulsive Cognitions Working, 2003), which assesses beliefs thought to play a role in the development of OCD symptoms, were positively associated with anxiety about H1N1 Swine Flu. Notably, obsessive beliefs are not necessarily specific to OCD, as they seem to contribute to multiple conditions involving pathological anxiety (Tolin et al., 2006). Although replication is needed, it appears that these beliefs also are related to anxiety during pandemic threats.

In addition, several studies have investigated whether beliefs and interpretations related to contamination are relevant to anxiety about pandemic illnesses. The Contamination Cognitions Scale (Deacon \& Olatunji, 2007) is a self-report inventory that assesses individuals' tendency to overestimate the likelihood and severity of negative consequences from touching objects that could be contaminated. Several studies have found that individuals with higher scores on this scale have increased anxiety in response to pandemic illnesses, including H1N1 Swine Flu (Wheaton et al., 2012), Zika virus (Blakey \& Abramowitz, 2017), and Ebola (Blakey et al., 2015).

Finally, threat-related appraisals of illness severity and controllability were related to worry about H1N1 in a large community sample (Scherr et al., 2017). Combined, these results suggest that individuals' pre-existing beliefs, as well as how they interpret contamination and the pandemic threat itself, contribute to anxiety responses. This is consistent with a cognitive model, and importantly, these cognitive variables might be targeted in therapy to relieve excessive anxiety.

\section{Transdiagnostic Cognitive Process Variables}

In addition to belief-based cognitions, several transdiagnostic process variables (which contribute to a range of psychological conditions) have also been investigated as potential factors in anxiety responses in past pandemics, including intolerance of uncertainty, disgust sensitivity, body vigilance, and anxiety sensitivity. 
Intolerance of uncertainty (IU) is defined as the "dispositional incapacity to endure the aversive response triggered by the perceived absence of salient, key, or sufficient information, and sustained by the associated perception of uncertainty" (Carleton, 2016a) and has been conceptualized as a transdiagnostic contributing factor to many anxiety-related conditions, including OCD and health anxiety. IU has been considered a relatively broad construct representing cognitive, emotional, and behavioral reactions to uncertainty in everyday life situations (Freeston et al., 1994). Contemporary models consider IU to be an important transdiagnostic factor contributing to multiple anxiety-related conditions (Carleton, 2016a, 2016b). A past study found that anxiety in response to the 2009 H1N1 pandemic was linked to higher IU (Taha et al., 2014). In addition, although somewhat different from the recent pandemics discussed above, difficulty tolerating uncertainty has also been noted as a factor in excessive fear of contracting HIV (Scragg, 1995). Difficulty facing the unknown might be an especially important contributing factor to pandemic fears during the early stages of disease outbreaks, when the extent of the threat the virus poses remains unclear. For individuals high in IU, ambiguity about how the pandemic will unfold may be highly anxiety-provoking. Importantly, IU has been described as a contributing factor to both OCD and health anxiety (Carleton, 2016a).

Another transdiagnostic process is disgust sensitivity, the propensity to experience disgust across situations. It has been linked to OCD symptoms, particularly those relating to contamination. Several studies reported that disgust sensitivity was associated with concerns about past pandemic threats in undergraduate students in the USA, including the H1N1 Swine Flu (Brand et al., 2013; Wheaton et al., 2012) and Ebola (Blakey et al., 2015). However, another study in undergraduate students found that disgust sensitivity was not a significant predictor of anxiety in response to the Zika virus (Blakey \& Abramowitz, 2017). These mixed results merit further research into whether disgust sensitivity might be relevant to fears of COVID-19.

Anxiety sensitivity refers to the tendency to fear body sensations associated with anxious arousal because of their perceived physical, psychological, or social consequences (Reiss, 1985). It is an additional transdiagnostic factor postulated to play a role in both health anxiety and OCD. Several studies in American undergraduates linked anxiety sensitivity to responses to pandemic threats, including H1N1 (Brand et al., 2013; Wheaton et al., 2012) and Ebola (Blakey et al., 2015), though another study found that it did not predict anxiety in response to the Zika virus (Blakey \& Abramowitz, 2017).

Another psychological process related to health anxiety is body vigilance, the tendency to vigilantly monitor the body for signs and symptoms of illness. Body vigilance has been linked to excessive anxiety in response to other communicable illnesses, including fears of HIV (Miller et al., 1988). It was also found to be significantly correlated with fears of some past pandemics among American undergraduate students, including Ebola (Blakey et al., 2015). However, body vigilance did not predict anxiety in response to H1N1 Swine Flu (Wheaton et al., 2012) or the Zika virus (Blakey \& Abramowitz, 2017). Thus, it is important for research to determine whether the tendency to attend to one's body for signs of illness may be relevant during COVID-19.

\section{Social Factors}

An important part of the diathesis-stress model is the perception of how one's social environment exerts a significant influence in the way individuals respond to pandemics. 
Specifically, perceived social support and perceived loneliness were two factors associated with psychological distress during the SARS pandemic.

A model derived from a Hong Kong-based investigation identified perceived social support from medical staff and family/friends as predictors, affecting SARS survivors' mental health with self-care and self-efficacy as mediators. The long-term psychological adjustment of SARS survivors was improved by perceived social support, yet perceived family/friend support showed a relatively stronger effect than perceived medical support (Mak et al., 2009).

In Toronto (one of the epicenters of the outbreak), patients infected with SARS reported experiencing feelings of loneliness and boredom, which were linked to psychological symptoms, such as anxiety about fever (Maunder et al., 2003). However, social isolation and disrupted contact with colleagues (due to recommended quarantine guidelines) were found to mediate the traumatic impact from the SARS outbreak on level of distress, in a sample of hospital workers (Maunder et al., 2004). In fact, a systematic thematic review on the adjustment of healthcare workers during the SARS outbreak found that social support was the most effective mechanism for minimizing future psychological complications (Brooks et al., 2018).

\section{Environmental/Situational Variables}

In addition to belief-based cognitions, transdiagnostic process variables, and social environment factors, certain environmental/situational factors increase the likelihood of developing pandemic-related anxiety. Some of these variables that have been implicated in past pandemic studies include working as a frontline worker, proximity to the epicenter, and information consumption.

The "bull's eye" model of disaster posits that distress is stronger the closer one is to the center of the disaster (Marshall et al., 2007). Consistent with this model, direct exposure to the outbreak, being quarantined, and having a relative or friend contract SARS were positively associated with posttraumatic stress symptoms 3 years after the crisis ended (Wu et al., 2009). On the other hand, personal level of exposure to SARS was not a primary determinant of anxiety, and closeness to the center of the epidemic was negatively related to anxiety levels (Xie et al., 2011).

In one of the few published reports to assess the role that direct exposure to a pandemic disease has on potential psychological and behavioral reactions, one study compared 25 individuals infected by Swine Flu to 25 individuals who were not infected by the virus (Goodwin et al., 2009). The impacted group experienced significantly higher levels of stress in comparison to the non-impacted group. Similarly, in a sample in India, Sahni et al. (2016) compared individuals who contracted H1N1 to matched controls and found elevated anxiety and fear of death among those who were infected. These results suggest that personal experiences with infection by a pandemic can cause significant distress.

Healthcare workers are on the "front lines," and are also likely to have first-hand experience with pandemic threats. Therefore, understanding their psychological response to outbreaks is important. Among those working in hospitals during SARS, one study reported that $75.3 \%$ experienced psychiatric symptoms (Chong et al., 2004), including anxiety, worry, depression, poor family relationships, and sleep problems. Some healthcare workers also reported compulsive checking of their body temperature 
and fear of spreading the illness to their families (Chong et al., 2004). Similarly, high rates of fear of the contagion and of infecting family members were found among staff in an independent sample of hospital workers during SARS (Maunder et al., 2003). Perhaps not surprisingly, Maunder et al. (2004) reported that health-related stress and anxiety were greater among healthcare workers who had direct contact with patients infected with SARS as compared to those who did not.

In addition to epicenter proximity, contracting the disease and working on the "front lines" with infected patients, media portrayals of pandemic threats also can impact individuals' anxiety levels. For example, Tausczik et al. (2012) analyzed online webtraffic and blog posts via linguistic analyses and found that usage of anxiety-related words linked to the H1N1 Swine Flu significantly increased public social media posts directly following newspaper article coverage of the virus. Similarly, consumption of news media reports was associated with anxiety about other pandemic illness threats, including the 2015-2016 Zika virus (Blakey \& Abramowitz, 2017), SARS (Xie et al., 2011) and Ebola (Blakey et al., 2015; Thompson et al., 2017) outbreaks. These data suggest that even for individuals who are fortunate enough not to directly encounter a pandemic illness (i.e., via infection or through work as a healthcare provider), media portrayals may serve as an important environmental factor contributing to pandemicrelated anxiety.

\section{Summary and Limitations of Existing Studies}

There are several notable limitations to the studies reviewed above. First, some of these studies were conducted with undergraduate subjects (Blakey \& Abramowitz, 2017; Blakey et al., 2015; Brand et al., 2013; Wheaton et al., 2012), and their results may not generalize to the broader population. Second, in the two studies conducted on Zika and Ebola fears, the investigations were carried out in the USA where there were only a handful of Zika cases and one Ebola case (Blakey \& Abramowitz, 2017; Blakey et al., 2015). Third, these studies tended to rely on self-report measures (Blakey \& Abramowitz, 2017; Blakey et al., 2015; Chong et al., 2004; Goodwin et al., 2009; Wheaton et al., 2012) and most utilized cross-sectional methods, which precluded causal inferences. In addition, to date, only a rather limited number of cognitive variables were investigated. Additional factors, such as, ruminative response styles, hopelessness and self-efficacy beliefs have not sufficiently been studied in past pandemics but may be relevant for understanding responses to COVID-19. There is evidence that the looming cognitive style (LCS) plays a significant role as a vulnerability factor for multiple forms of anxiety (Adler \& Strunk, 2010; Riskind et al., 2000; Yeo et al., 2020). This maladaptive cognitive style may be particularly relevant to anxiety about pandemic illnesses, but no study has yet tested this potential association, necessitating future research. Finally, these studies often did not consider how responses to pandemics may differ according to race, age, gender, or socioeconomic status. This is particularly important in that the majority of responses in undergraduate survey studies have come from largely non-Hispanic white participants (Blakey \& Abramowitz, 2017; Blakey et al., 2015).

Notwithstanding these notable limitations, these existing studies provide important information about factors associated with pandemic-related anxiety. Specifically, 
anxiety during past pandemics has been linked to beliefs and interpretations, transdiagnostic cognitive processes, social factors, and environmental/situational variables. These factors can be thought of in terms of a diathesis-stress model in which people with certain vulnerability factors (cognitions, beliefs, and transdiagnostic processes) interact with proximal factors (e.g., perceived social support, isolation, infection, proximity to the disease epicenter, working in healthcare on the front lines, and media exposure) and thereby result in pathological pandemic-related anxiety. Although past pandemics provide an informative model for factors that increase pandemic-related anxiety, the COVID-19 pandemic is also unique in many respects, as discussed below.

\section{How COVID-19 Differs From Other Recent Pandemics}

There are several important factors differentiating COVID-19 from the abovementioned pandemics, which may affect its psychological impact. Although several characteristics of COVID-19 may be linked to the development of a broad set of psychological symptoms, we focus on factors relevant to contamination and health anxiety.

\section{Disease Factors}

COVID-19 is a highly contagious disease that is now thought to be transmitted through multiple pathways including droplets, aerosols, and even fecal matter (Huang et al., 2020). One study reported that it was transmitted through a restaurant air conditioning system (Lu et al., 2020). Modeling the rate of transmission showed an exponential growth rate (Kucharski et al., 2020), depending on multiple factors including population density and carrying out mitigation efforts (e.g., social distancing, mask-wearing, sterilizing, testing, contract tracing). Factors that seem to be contributing to the high level of contagiousness include the fact that up to one quarter of infected people are asymptomatic, but nonetheless can infect others (Bai et al., 2020). Toward the end of 2020, variants of COVID 19 emerged, including some with even higher transmissibility rates (Galloway et al., 2021). At the same time, the virus has been shown to be highly resilient (Goh et al., 2020), lasting a relatively long time in saliva and other fluids outside the body, with the possibility of transmission via multiple shedding routes (Wang, Hu, et al., 2020a).

Although other diseases may be as contagious as COVID-19, many are not as lethal. While COVID-19's level of lethality is lower than SARS (World Health Organization, 2020), it is greater than Swine Flu. In the Wuhan Province, analysis of 339 consecutive cases admitted to a hospital showed that over two-thirds were severe or critical. Among those hospitalized cases, in the sample over age 60 (mean age $=71 \pm 8$ ), the death rate was $19.5 \%$ (Wang, Pan, et al., 2020b). Modeling in China of the case fatality ratio showed an overall rate of $1.4 \%$ with death rates increasing with age. Depending on disease (e.g., viral load, disease strain) and other risk factors (e.g., age, health conditions), individuals contracting COVID-19 face the possibility of a severe course or even death.

In addition to the speed of transmission, rapid course, and relatively high death rate, two of the common symptoms of COVID-19 are similar to symptoms of anxiety. Notably, typical COVID-19 symptoms can include shortness of breath and persistent 
chest pressure or pain (CDC, 2020), even in those with milder disease courses. The similarity of these physical symptoms to the physical sensations common in panic attacks may cause uncertainty as individuals high in anxiety sensitivity question whether their anxiety symptoms are due to COVID-19 infection.

Additionally, as COVID-19 is a new disease, tools that we have in our arsenal to fight other diseases are not available. Although there are medical interventions that can support respiration and immune functioning, an empirically supported and targeted treatment has not been found. Combined, the lack of adequate testing and the absence of an effective treatment heighten the disease threat level and contribute to the overall uncertainty about COVID-19 which, in turn, increase health anxiety.

Another factor distinguishing COVID-19 from other pandemics is the level of news coverage it has received. In particular, news reports described shortages of public health resources to treat cases including hospital beds, ventilators, and in some areas personal protective equipment (PPE) for both hospital workers and others treating sick patients (Emanuel et al., 2020). Furthermore, as hospital beds filled with COVID-19 patients, resources for other medical disorders were taxed, with hospital administrators scrambling to organize their approaches to both COVID-19 and other conditions. In some cities, field hospitals in convention centers, parks, and other spaces were brought on line, underscoring the seriousness of the threat.

In developing countries, the effects of COVID-19 may be particularly impactful, especially in countries that have poor healthcare infrastructure (Hopman et al., 2020). Even within Westernized countries, individuals from lower socioeconomic statuses may be particularly at risk during COVID-19. The virus has also had a differential impact on communities of color in the USA, with an infection rate that is three times higher in 131 predominantly black counties than that of predominantly white counties and a death rate that is six times higher (Yancy, 2020). Health comorbidities alone likely do not explain this discrepancy; rather, other factors such as high housing density, poor access to healthy foods, and the lack of work-at-home options are all contributing factors to consider.

\section{Behavioral Factors}

In the absence of other tools to fight COVID-19, unprecedented social distancing has been recommended to curb its spread, in countries such as the USA, Germany, and South Korea but not in Sweden. Compliance with advice to maintain social distance (at least $6 \mathrm{ft}$ from others) may be easier for some than for others. Many people must take public transportation, enter crowded buildings, or otherwise must come into contact with strangers of unknown health status on a daily basis. Because their jobs or circumstances mandate this degree of close interaction, people continue to function with full knowledge that they may be exposed to the virus. Moreover, fully maintaining social distance is impossible for most people as they still need to obtain food, medicine, and other necessities. Furthermore, while some care can be delivered effectively via telemedicine, many medical and medical testing appointments require patients to see providers in person. Similarly, although many mental health professionals have moved their practices to teletherapy platforms, this has not been done universally, leaving many existing and prospective patients without needed services, including among those receiving treatment for OCD and SHA. 
During the beginning of the pandemic, as concern about the contagiousness of COVID-19 increased, public health authorities encouraged decontamination strategies ranging from hand washing to surface sanitizing of most objects that enter peoples' homes. Rituals formerly considered "OCD" like wiping groceries and other packages with germ-killing solutions became normative. Complicating this issue, there were shortages of anti-bacterial wipes and other disinfecting products.

In the era of the 24-hour news cycle, two factors related to information dissemination could potentially cause increased anxiety. First, people have the option of constantly watching coverage of COVID-19 on television or reading about it in the internet. For many, frequent exposure to news reporting of dire circumstances can increase anxiety (Asmundson \& Taylor, 2020) and in response additional checking, with the anxiety and checking alternating to create a downward spiraling effect (Asmundson \& Taylor, 2020). Some have even studied the phenomenon in which seeking health information online can paradoxically increase fears of becoming ill, the so named construct of "cyberchondriasis" (Starcevic \& Berle, 2013). Second, with the democratization of the internet, articles widely available for reading may not have undergone sufficient content review, leading to the proliferation of "fake" news on some outlets. Whereas 100 years ago, during the Spanish Flu epidemic, the only news source was print news. Now, people can obtain information on the COVID-19 pandemic from hundreds of TV and internet sources, many of which had not been vetted. Reading unreliable news sources can lead to an increasing sense of uncertainty, as people are unsure what to believe.

\section{Social Factors}

Before the COVID-19 pandemic occurred, most individuals residing in locales with no history of past pandemic exposure had never heard of "social distancing" or worn masks in the normal course of their lives. In the span of a few weeks, schools from pre$\mathrm{K}$ through college and graduate levels migrated to distance learning platforms. Cultural centers, performance venues, retail stores, and businesses other than those considered necessary, such as pharmacies and grocery stores, were shuttered. As people undergo weeks of "stay at home" orders, tensions within already tense family situations may be exacerbated. Individuals living together may have different disease statuses, leading to challenges in preventing the healthier residents from becoming sick. Even if there is not notable illness in the household, if one or more members is forced to interact with others outside due to their job or some other circumstance, there is risk that other family members or housemates may be unknowingly infected. Indeed, differing approaches to disease mitigation within cohabitating families may affect anxiety levels. For others who live alone, the level of isolation may be great. They may not have anyone to take care of them if they become ill or obtain for them medical supplies or even food. Furthermore, for people in isolation sources of social support for coping with health and anxiety may be less readily available. On the other hand, in some communities, citizens do not have the same privilege of being able to "socially distance," resulting in greater anxiety about infection exposure. How individuals are affected by these factors varies greatly, depending on their particular circumstances (e.g., if they live in a locale where a stay-at-home order is in place, if they had to forego important activities or events, if they live alone, or if they live in families where there is one or 
more infected individuals or more extensive outside contact). Already, data are emerging while the COVID-19 pandemic unfolds, suggesting that perceived social support and loneliness may be critical factors to how individuals are able to cope with COVID19 (Bareket-Bojmel et al., 2021). Thus, the social disruption caused by COVID-19 makes it stand apart from past pandemics and warrants particular attention in understanding how the population responds.

\section{Economic factors}

In modern history, no other disease event has had a more rapid and severe effect on global markets, trickling down to many industries and businesses, large and small, as well as the labor market. Although economic changes have varied widely depending on each country's strategy of curbing the virus, in many countries, including the USA, individuals have seen the value of their retirement savings significantly diminish in a short amount of time, while many others have lost their jobs, resulting in the highest unemployment rate since the Great Depression. Entire industries, most notably in the hospitality field (travel, tourism, and restaurants), have been especially hard-hit as have other businesses in their orbits. Reduced financial resources may affect peoples' ability to pay for health insurance, medications, or medical care. However, in countries that have not ordered businesses to close such as Taiwan, Denmark, and Sweden, their economies have been less impacted. Although federal legislation efforts in the USA and other nations may provide varying levels of financial relief, considering the global market system, the longer term economic effects of COVID-19 are highly uncertain. Although the pandemic continues to unfold, rapidly emerging data suggest anxiety about the economic consequences of COVID-19 is essentially equal to anxiety about its health consequences (Bareket-Bojmel et al., 2020). COVID-19 is unique in its widespread economic consequences, and anxiety in this regard also deserves research attention in understanding the pandemic's widespread effects.

\section{Country-Specific Response to COVID-19}

Although COVID-19 is a global pandemic affecting nearly all nations, each country has responded to the pandemic in a different manner. For instance, Taiwan acted quickly to try and diminish the spread of the disease by proactively seeking out people with respiratory agitation, tracking individuals on their cell phones to ensure that those put in quarantine abided by it, and setting up hotlines for citizens to report symptoms (Wang, Ng, \& Brook, 2020c). These efforts have limited disease spread and the number of deaths in Taiwan. Sweden has let their schools and businesses remain open and has not enacted strict "social distancing" policies (Rolander, 2020). New Zealand has stood out by adopting one of the strictest and earliest lockdowns in the world, with a very low number of deaths (Brockett, 2020). These wide-ranging responses as well as the varied severity of the outbreak will likely have differing effects on those with OCD and health anxiety across countries. Specifically, in addition to nation-specific differences in COVID-19 mortality, the various ways that governments responded could modulate anxiety responses. Some governments appeared to downplay the threat, others promoted cohesive responses of shared communal responsibilities, and others lapsed into mixed and confusing messages. Additionally, media outlets have presented information 
on COVID-19 differently, which would most likely impact the way individuals respond psychologically to the pandemic. Future research is needed to determine the ways in which government responses and media portrayals may have led to adaptive versus maladaptive behavioral responses among the public.

\section{COVID-19 Impact on Those with Contamination Fears}

As noted, COVID-19 has a high transmission rate and can survive on surfaces for longer than other viruses. Because of the disease's resiliency, many individuals with contamination fears will experience increased anxiety and distress over acquiring this disease. Furthermore, many carriers of COVID-19 are asymptomatic, potentially leading to a fear of "everyone" and not just those who are visibly ill. Living with someone who goes into public and is in close proximity to others may also increase contamination fears. Typically, contamination-fearful individuals cope with their fears by taking sanitary measures such as washing hands, using bleach, and wearing masks, compulsions that can actually worsen the problem. However, in the current situation, many communities have experienced shortages of cleaning supplies, hand sanitizer, and masks, which may exacerbate the levels of contamination distress. Further complicating the problem is the level of uncertainty over where the virus actually is, whether it is brought into the house, which surfaces need to be cleaned, and to what extent.

In addition to the concern about the perceived threat to one's own life, some with contamination-related OCD are more worried about the threat to others, and may even have an exaggerated sense of responsibility. Messaging about social distancing underscores the responsibility everyone has for keeping other people from getting sick. In addition, COVID-19 may be transmitted via asymptomatic carriers through unwitting contamination. All these factors may increase fears of spreading the disease to others, as commonly reported in those with OCD symptoms motivated by fears of being responsible for harm (McKay et al., 2004). Fear of being responsible for a loved one contracting COVID-19 would likely be a potent trigger for these individuals.

\section{COVID-19 Impact on Those with Health Anxiety}

Similar to contamination anxiety, those with health anxiety will probably feel a rise in anxiety linked to COVID-19. Likewise, as health anxiety and other more specific anxiety constructs have been linked to fears of other pandemics, we would predict that people with similar anxiety profiles would become more fearful of COVID-19.

COVID-19 is highly transmittable, and has a rapid course and high mortality rate, factors which, considered together, increase the threat of the disease and fuel feelings of anxiety. A few symptoms of COVID-19 are highly similar to anxiety symptoms, leading to uncertainty as to what is their cause. Many individuals with health anxiety compulsively check their body temperature to ensure that they are not sick, yet many individuals with COVID-19 do not exhibit symptoms. These individuals with a predilection toward fear of illness may not have their distress alleviated by engaging in the compulsion of temperature checking as there is still a possibility that they have this disease. Moreover, mandated social distancing has decreased access to healthcare, 
as several medical practices have limited or shuttered their in-person services. Similarly, psychotherapy to address anxiety is currently available only through telehealth in many locations, creating uncertainty about service provision and leaving anxiety untreated as patients contemplate seeing a provider using an unfamiliar modality (e.g., videoconferencing or telephones). Furthermore, for those living alone, social distancing may also mean that there is no one to take care of them if they get sick, as family may be reluctant to expose themselves to the virus in order to help with care. People who have lost jobs or seen decreased income may fear being unable to afford health insurance or pay other costs associated with securing medical attention. Further exacerbating these concerns are media reports. Although much of the press on COVID19 has been helpful in educating the public, other reports convey false information, leading to mixed messaging and a sense of uncertainty regarding what is the truth about the disease. Media coverage of diseases can increase health anxiety in some people (Asmundson \& Taylor, 2020). Given this finding and knowing that health anxiety is one of several psychological factors that can influence the way someone responds to a virus (Taylor, 2019), it would be hypothesized that individuals susceptible to health anxiety will experience more severe symptoms with increased exposure to media covering COVID-19. For those prone to seek assurance from information sources, the sheer volume of available information provides an opportunity for excessive news consumption, which could become a form of compulsive checking.

\section{Summary and Discussion}

There is relatively little empirical research into the psychological impacts of pandemic disease outbreaks. Although few in number, these studies do show that pandemics can have substantial psychological effects. Based on this existing literature, is it likely that COVID-19 will have a significant mental health impact which may be particularly acute among individuals with OCD, health anxiety, and individuals in certain environments such as healthcare workers. Factors specific to COVID-19 suggest that this outbreak may have particularly strong psychological effects. Specifically, the COVID19 virus is associated with severe illness or death and is highly transmissible. These features would naturally elevate fears of contracting the illness. In addition, the fact that many individuals infected with COVID-19 do not show symptoms may be highly anxiety-provoking among individuals who are intolerant of uncertainty. Finally, prescribed behavioral changes (e.g., mandated social distances and requirements to wear masks in public) to reduce the likelihood of spreading the infection to others may be anxiety-provoking for individuals with inflated responsibility for causing harm, as they may appraise even momentary lapses in rule adherence as a substantial threat.

Together, these factors suggest that the COVID-19 outbreak may be especially taxing for individuals with pathological health anxiety and OCD. At the same time, quarantine restrictions and fears of the disease may interfere with these individuals' ability to get treatment. With amazing speed, many psychotherapy and psychiatry providers have transitioned to telehealth delivery (delivering treatment via phone and videoconferencing). Fortunately, there is an emerging evidence-base for the effectiveness of remote therapy for OCD and anxiety (Backhaus et al., 2012; Bee et al., 2008; Wootton, 2016). Yet, future research is going to be needed to determine how best to 
accomplish this modality of treatment in light of illness outbreaks such as COVID-19. Specifically, exposure-based psychotherapies are frequently effective for treating anxiety disorders and OCD, and yet exposure is not practical when there is a realistic danger. Therefore, clinicians will need to adapt their practices in light of delivering therapy during a pandemic quarantine. For example, imaginal exposure techniques might allow patients to confront feared consequences in a realistically safe way.

Given the lack of past research on cultural and socioeconomic factors related to the mental health impact of pandemics, it is important to consider how COVID-19 will affect individuals from different backgrounds. For instance, the way people from different cultures respond to health crises may depend upon contextual factors including views of the disease, availability of telepsychology resources, and mental health stigma. Individuals from low socioeconomic strata may have a particularly challenging time during the COVID-19 pandemic as they will likely suffer from greater economic hardships and may also lack the technological resources to access treatment digitally.

The significant impact of the COVID-19 outbreak will require substantial effort on behalf of both clinicians and researchers to be able to effectively assess and treat pathological anxiety in response to the outbreak. Although the challenge will be great, this opportunity and the lessons learned from it may allow the mental health field to improve our ability to address similar challenges in the future.

\section{Declarations}

Conflict of Interest The authors declare no competing interests.

\section{References}

Abramowitz, J. S., \& Braddock, A. E. (2006). Hypochondriasis: Conceptualization, treatment, and relationship to obsessive-compulsive disorder. The Psychiatric Clinics of North America, 29(2), 503-519. https:// doi.org/10.1016/j.psc.2006.02.008.

Adler, A. D., \& Strunk, D. R. (2010). Looming maladaptive style as a moderator of risk factors for anxiety. Cognitive Therapy and Research, 34, 59-68. https://doi.org/10.1007/s10608-008-9221.

Asmundson, \& Taylor, S. (2020). Coronaphobia: Fear and the 2019-nCoV outbreak. Journal of Anxiety Disorders, 70, 102196. https://doi.org/10.1016/j.janxdis.2020.102196.

Asmundson, Abramowitz, J. S., Richter, A. A., \& Whedon, M. (2010). Health anxiety: Current perspectives and future directions. Current Psychiatry Reports, 12(4), 306-312. https://doi.org/10.1007/s11920-0100123-9.

American Psychiatric Association. (2013). Diagnostic and statistical manual of mental disorders (5th ed.). https://doi.org/10.1176/appi.books.9780890425596

Backhaus, A., Agha, Z., Maglione, M. L., Repp, A., Ross, B., Zuest, D., ... Thorp, S. R. (2012). Videoconferencing psychotherapy: A systematic review. Psychological Services, 9(2), 111-131. doi: https://doi.org/10.1037/a0027924

Bai, Y., Yao, L., Wei, T., Tian, F., Jin, D. Y., Chen, L., \& Wang, M. (2020). Presumed asymptomatic carrier transmission of COVID-19. JAMA. https://doi.org/10.1001/jama.2020.2565.

Bareket-Bojmel, L., Shahar, G., \& Margalit, M. (2020). COVID-19-related economic anxiety is as high as health anxiety: Findings from the USA, the UK, and Israel. International Journal of Cognitive Therapy, 1-9. doi:https://doi.org/10.1007/s41811-020-00078-3

Bareket-Bojmel, L., Shahar, G., Abu-Kaf, S., \& Margalit, M. (2021). Perceived social support, loneliness, and hope during the COVID-19 pandemic: Testing a mediating model in the UK, USA, and Israel. The British Journal of Clinical Psychology. https://doi.org/10.1111/bjc.12285.

Beck, A. T. (1979). Cognitive therapy and the emotional disorders. Penguin. 
Bee, P. E., Bower, P., Lovell, K., Gilbody, S., Richards, D., Gask, L., \& Roach, P. (2008). Psychotherapy mediated by remote communication technologies: A meta-analytic review. BMC Psychiatry, 8, 60. https:// doi.org/10.1186/1471-244X-8-60.

Blakey, S. M., \& Abramowitz, J. S. (2017). Psychological predictors of health anxiety in response to the Zika virus. Journal of Clinical Psychology in Medical Settings, 24(3-4), 270-278. https://doi.org/10.1007/ s10880-017-9514-y.

Blakey, S. M., Reuman, L., Jacoby, R. J., \& Abramowitz, J. S. (2015). Tracing "Fearbola": Psychological predictors of anxious responding to the threat of Ebola. Cognitive Therapy and Research, 39(6), 816-825.

Brand, J., McKay, D., Wheaton, M. G., \& Abramowitz, J. S. (2013). The relationship between obsessive compulsive beliefs and symptoms, anxiety and disgust sensitivity, and Swine Flu fears. Journal of Obsessive-Compulsive and Related Disorders, 2(2), 200-206.

Brockett, M. (2020). How one country set a course to totally eliminate the COVID-19 virus. Fortune. Retrieved from https://fortune.com/2020/04/18/new-zealand-jacinada-ardern-eliminate-coronavirus/. Accessed 20 Apr 2020.

Brooks, S. K., Dunn, R., Amlot, R., Rubin, G. J., \& Greenberg, N. (2018). A systematic, thematic review of social and occupational factors associated with psychological outcomes in healthcare employees during an infectious disease outbreak. Journal of Occupational and Environmental Medicine, 60(3), 248-257. https://doi.org/10.1097/JOM.0000000000001235.

Carleton, R. N. (2016a). Fear of the unknown: One fear to rule them all? Journal of Anxiety Disorders, 41, 521. https://doi.org/10.1016/j.janxdis.2016.03.011.

Carleton, R. N. (2016b). Into the unknown: A review and synthesis of contemporary models involving uncertainty. Journal of Anxiety Disorders, 39, 30-43. https://doi.org/10.1016/j.janxdis.2016.02.007.

CDC. (2014). Outbreaks chronology: Ebola virus disease. Retrieved from http://www.cdc.gov/vhf/ebola/ outbreaks/index.html. Accessed 30 Apr 2020.

CDC. (2016). Zika Virus. Retrieved from http://www.cdc.gov/zika/about/questions.html.

CDC. (2020). Coronavirus. Retrieved from https://www.cdc.gov/coronavirus/2019-ncov/index.html. Accessed 30 Apr 2020.

Chong, M. Y., Wang, W. C., Hsieh, W. C., Lee, C. Y., Chiu, N. M., Yeh, W. C., . . Chen, C. L. (2004). Psychological impact of severe acute respiratory syndrome on health workers in a tertiary hospital. British Journal of Psychiatry, 185, 127-133. doi:https://doi.org/10.1192/bjp.185.2.127

Wuhan Municipal Health Commission (2019). Report of clustering pneumonia etiology in Wuhan City. Retrieved from http://wjw.wuhan.gov.cn/front/web/showDetail/2019123108989

Deacon, B., \& Abramowitz, J. S. (2008). Is hypochondriasis related to obsessive-compulsive disorder, panic disorder, or both? An empirical evaluation. Journal of Cognitive Psychotherapy, 22(2), 115-127.

Deacon, B., \& Olatunji, B. O. (2007). Specificity of disgust sensitivity in the prediction of behavioral avoidance in contamination fear. Behaviour Research and Therapy, 45(9), 2110-2120. https://doi.org/ 10.1016/j.brat.2007.03.008.

Deeks, S. G., Overbaugh, J., Phillips, A., \& Buchbinder, S. (2015). HIV infection. Nature Reviews Disease Primers, 1, 15035. https://doi.org/10.1038/nrdp.2015.35.

Emanuel, E. J., Persad, G., Upshur, R., Thome, B., Parker, M., Glickman, A., . . Phillips, J. P. (2020). Fair allocation of scarce medical resources in the time of Covid-19. New England Journal of Medicine doi: https://doi.org/10.1056/NEJMsb2005114

Freeston, M. H., Rhéaume, J., Letarte, H., Dugas, M. J., \& Ladouceur, R. (1994). Why do people worry? Personality and Individual Differences, 17(6), 791-802.

Galloway, S. E., Paul, P., MacCannell, D. R., Johansson, M. A., Brooks, J. T., MacNeil, A., ... Dugan, V. G. (2021). Emergence of SARS-CoV-2 B.1.1.7 lineage-United States, December 29, 2020-January 12, 2021. MMWR Morbidity and Mortality Weekly Report, 70(3), 95-99. doi:https://oi.org/10.15585/mmwr. $\mathrm{mm} 7003 \mathrm{e} 2$

Goh, G. K., Dunker, A. K., Foster, J. A., \& Uversky, V. N. (2020). Shell disorder analysis predicts greater resilience of the SARS-CoV-2 (COVID-19) outside the body and in body fluids. Microbial Pathogenesis, 144, 104177. https://doi.org/10.1016/j.micpath.2020.104177.

Goodwin, R., Haque, S., Neto, F., \& Myers, L. B. (2009). Initial psychological responses to Influenza A, H1N1 ("Swine flu”). BMC Infectious Diseases, 9, 166. https://doi.org/10.1186/1471-2334-9-166.

Hills, S. L., Russell, K., Hennessey, M., Williams, C., Oster, A. M., Fischer, M., \& Mead, P. (2016). Transmission of Zika virus through sexual contact with travelers to areas of ongoing transmissionContinental United States, 2016. MMWR. Morbidity and Mortality Weekly Report, 65(8), 215-216. https://doi.org/10.15585/mmwr.mm6508e2.

Hopman, J., Allegranzi, B., \& Mehtar, S. (2020). Managing COVID-19 in low- and middle-income countries. JAMA. https://doi.org/10.1001/jama.2020.4169. 
Huang, C., Wang, Y., Li, X., Ren, L., Zhao, J., Hu, Y.,... Cao, B.(2020). Clinical features of patients infected with 2019 novel coronavirus in Wuhan, China. Lancet, 395(10223), 497-506. https://doi.org/10.1016/ S0140-6736(20)30183-5.

Johnson, N. P., \& Mueller, J. (2002). Updating the accounts: Global mortality of the 1918-1920 "Spanish" influenza pandemic. Bulletin of the History of Medicine, 105-115.

Jones, M. K., \& Menzies, R. G. (1997). The cognitive mediation of obsessive-compulsive handwashing. Behaviour Research and Therapy, 35(9), 843-850. https://doi.org/10.1016/s0005-7967(97)00035-1.

Kucharski, A. J., Russell, T. W., Diamond, C., Liu, Y., Edmunds, J., Funk, S., . . Centre for Mathematical Modelling of Infectious Diseases, COVID-19 working group. (2020). Early dynamics of transmission and control of COVID-19: A mathematical modelling study. Lancet Infectious Diseases. doi:https://doi.org/ 10.1016/S1473-3099(20)30144-4

Lu, J., Gu, J., Li, K., Xu, C., Su, W., Lai, Z., . . Yang, Z. (2020). COVID-19 outbreak associated with air conditioning in restaurant, Guangzhou, China, 2020. Emerging Infectious Diseases, 26(7). doi:https://doi. org/10.3201/eid2607.200764

Mak, W. W., Law, R. W., Woo, J., Cheung, F. M., \& Lee, D. (2009). Social support and psychological adjustment to SARS: The mediating role of self-care self-efficacy. Psychology \& Health, 24(2), 161-174. https://doi.org/10.1080/08870440701447649.

Marshall, R. D., Bryant, R. A., Amsel, L., Suh, E. J., Cook, J. M., \& Neria, Y. (2007). The psychology of ongoing threat: Relative risk appraisal, the September 11 attacks, and terrorism-related fears. The American Psychologist, 62(4), 304-316. https://doi.org/10.1037/0003-066X.62.4.304.

Maunder, Hunter, J., Vincent, L., Bennett, J., Peladeau, N., Leszcz, M., ..., Mazzulli, T. (2003). The immediate psychological and occupational impact of the 2003 SARS outbreak in a teaching hospital. CMAJ, 168(10), 1245-1251. Retrieved from https:/www.ncbi.nlm.nih.gov/pubmed/12743065

Maunder, Lancee, W. J., Rourke, S., Hunter, J. J., Goldbloom, D., Balderson, K., ..., Fones, C. S. (2004). Factors associated with the psychological impact of severe acute respiratory syndrome on nurses and other hospital workers in Toronto. Psychosomatic Medicine, 66(6), 938-942. doi:https://doi.org/10.1097/ 01.psy.0000145673.84698.18

McKay, D., Abramowitz, J. S., Calamari, J. E., Kyrios, M., Radomsky, A., Sookman, D., ... Wilhelm, S. (2004). A critical evaluation of obsessive-compulsive disorder subtypes: Symptoms versus mechanisms. Clinical Psychology Review, 24(3), 283-313. doi:https://doi.org/10.1016/j.cpr.2004.04.003

Miller, D., Acton, T., \& Hedge, B. (1988). The worried well: Their identification and management. Journal of the Royal College of Physicians of London, 22, 158-165.

Murphy, D. L., Timpano, K. R., Wheaton, M. G., Greenberg, B. D., \& Miguel, E. C. (2010). Obsessivecompulsive disorder and its related disorders: A reappraisal of obsessive-compulsive spectrum concepts. Dialogues in Clinical Neuroscience, 12(2), 131-148 Retrieved from https://www.ncbi.nlm.nih.gov/ pubmed/20623919.

Obsessive Compulsive Cognitions Working Group. (2003). Psychometric validation of the Obsessive Beliefs Questionnaire and the Interpretation of Intrusions Inventory: Part I. Behaviour Research and Therapy, 41(8), 863-878. https://doi.org/10.1016/s0005-7967(02)00099-2.

Reiss S, McNally RJ. (1985) Expectancy model of fear. In S Reiss and RR Bootzin (Eds). Theoretical issues in behaviour therapy (107-121). San Diego: Academic Press.

Riskind, J. H. (1997). Looming vulnerability to threat: A cognitive paradigm for anxiety. Behaviour Research and Therapy, 35(8), 685-702. https://doi.org/10.1016/s0005-7967(97)00011-9.

Riskind, J. H., Williams, N. L., Gessner, T., Chrosniak, L. D., \& Cortina, J. (2000). The looming maladaptive style: Anxiety, danger, and schematic processing. Journal of Personality and Social Psychology, 79, 837852.

Rolander, N. (2020). Sweden says controversial virus strategy proving effective. Bloomberg. Retrieved from https://www.msn.com/en-us/news/world/sweden-says-controversial-virus-strategy-proving-effective/arBB12SFN0. Accessed 30 Apr 2020.

Sahni, S. P., Kumar, V., Srivastava, D., \& Jain, G. (2016). Psychological responses to influenza A H1N1 ("Swine Flu") in India. Journal of Psychosocial Research, 11(1), 201-213.

Scherr, C. L., Jensen, J. D., \& Christy, K. (2017). Dispositional pandemic worry and the health belief model: Promoting vaccination during pandemic events. Journal of Public Health (Oxford, England), 39(4), e242-e250. https://doi.org/10.1093/pubmed/fdw101.

Scragg, P. (1995). A critical analysis of morbid fear of HIV/AIDS. Clinical Psychology \& Psychotherapy, 2(4), 278-284.

Starcevic, V., \& Berle, D. (2013). Cyberchondria: Towards a better understanding of excessive health-related Internet use. Expert Review of Neurotherapeutics, 13(2), 205-213. https://doi.org/10.1586/ern.12.162. 
Taha, S., Matheson, K., Cronin, T., \& Anisman, H. (2014). Intolerance of uncertainty, appraisals, coping, and anxiety: The case of the 2009 H1N1 pandemic. British Journal of Health Psychology, 19(3), 592-605. https://doi.org/10.1111/bjhp.12058.

Tausczik, Y., Faasse, K., Pennebaker, J. W., \& Petrie, K. J. (2012). Public anxiety and information seeking following the H1N1 outbreak: Blogs, newspaper articles, and Wikipedia visits. Health Communication, 27(2), 179-185. https://doi.org/10.1080/10410236.2011.571759.

Taylor, S. (2019). The psychology of pandemics: Preparing for the next global outbreak of infectious disease. Cambridge Scholars Publishing.

Taylor, S., \& Asmundson. (2004). Treating health anxiety: A cognitive behavioral approach. New York. Guilford.

Taylor, S., McKay, D., \& Abramowitz, J. S. (2012). Hypochondriasis and health-related anxiety. In P. Sturmey \& M. Herson (Eds.), Handbook of evidence-based practice in clinical psychology (II ed., pp. 603-619). Adult disorders.

Thompson, R. R., Garfin, D. R., Holman, E. A., \& Silver, R. C. (2017). Distress, worry, and functioning following a global health crisis: A national study of Americans' responses to Ebola. Clinical Psychological Science, 5(3), 513-521.

Tolin, D. F., Worhunsky, P., \& Maltby, N. (2006). Are "obsessive" beliefs specific to OCD?: A comparison across anxiety disorders. Behaviour Research and Therapy, 44(4), 469-480. https://doi.org/10.1016/j. brat.2005.03.007.

Triana-Baltzer, G. B., Gubareva, L. V., Nicholls, J. M., Pearce, M. B., Mishin, V. P., Belser, J. A., ... Fang, F. (2009). Novel pandemic influenza A(H1N1) viruses are potently inhibited by DAS181, a sialidase fusion protein. PLoS One, 4(11), e7788. doi:https://doi.org/10.1371/journal.pone.0007788

Wang, Hu, B., Hu, C., Zhu, F., Liu, X., Zhang, J., ..., Peng, Z. (2020a). Clinical characteristics of 138 hospitalized patients with 2019 novel coronavirus-infected pneumonia in Wuhan, China. JAMA. doi: https://doi.org/10.1001/jama.2020.1585

Wang, C., Pan, R., Wan, X., Tan, Y., Xu, L., Ho, C. S., \& Ho, R. C. (2020b). Immediate psychological responses and associated factors during the initial stage of the 2019 coronavirus disease (COVID-19) epidemic among the general population in China. International Journal of Environmental Research and Public Health, 17(5), 1729. https://doi.org/10.3390/ijerph17051729.

Wang, C. J., Ng, C. Y., \& Brook, R. H. (2020c). Response to COVID-19 in Taiwan: Big data analytics, new technology, and proactive testing. JAMA. https://doi.org/10.1001/jama.2020.3151.

Watanabe, T., \& Kawaoka, Y. (2011). Pathogenesis of the 1918 pandemic influenza virus. PLoS Pathogens, 7(1), e1001218. https://doi.org/10.1371/journal.ppat.1001218.

Wheaton, M. G., Abramowitz, J. S., Berman, N. C., Fabricant, L. E., \& Olatunji, B. O. (2012). Psychological predictors of anxiety in response to the H1N1 (swine flu) pandemic. Cognitive Therapy and Research, $36(3), 210-218$.

Wootton, B. M. (2016). Remote cognitive-behavior therapy for obsessive-compulsive symptoms: A metaanalysis. Clinical Psychology Review, 43, 103-113. https://doi.org/10.1016/j.cpr.2015.10.001.

World Health Organziation (2020). Coronavirus disease 2019 (COVID-19): Situation report, 72.

Wu, P., Fang, Y., Guan, Z., Fan, B., Kong, J., Yao, Z., ... Hoven, C. W. (2009). The psychological impact of the SARS epidemic on hospital employees in China: Exposure, risk perception, and altruistic acceptance of risk. Canadian Journal of Psychiatry, 54(5), 302-311. doi:https://doi.org/10.1177/ 070674370905400504

Xie, X.-F., Stone, E., Zheng, R., \& Zhang, R.-G. (2011). The 'Typhoon Eye Effect': Determinants of distress during the SARS epidemic. Journal of Risk Research, 14(9), 1091-1107.

Yancy, C. W. (2020). COVID-19 and African Americans. JAMA. https://doi.org/10.1001/jama.2020.6548.

Yeo, G. C., Hong, R. Y., \& Riskind, J. H. (2020). Looming cognitive style and its associations with anxiety and depression: A meta-analysis. Cognitive Therapy and Research, 44, 445-467. https://doi.org/10.1007/ s10608-020-10089-1.

Publisher's Note Springer Nature remains neutral with regard to jurisdictional claims in published maps and institutional affiliations. 